\title{
A spinal epidural abscess due to Streptobacillus moniliformis infection following a rat bite: case report
}

\author{
*Alexander Hammer, MD, ${ }^{1}$ Dorit Wolff, MD, ${ }^{2}$ Walter Geißdörfer, PhD, ${ }^{2}$ Michael Schrey, MD, ${ }^{1}$ \\ Renate Ziegler, MD, ${ }^{3}$ Hans-Herbert Steiner, MD, ${ }^{1}$ and Christian Bogdan, MD²

\begin{abstract}
1Department of Neurosurgery, Paracelsus Medical University, Nürnberg; ${ }^{2}$ Mikrobiologisches Institut-Klinische Mikrobiologie, Immunologie und Hygiene, Friederich-Alexander-Universität Erlangen-Nürnberg, Universitätsklinikum Erlangen, Erlangen, Germany; and ${ }^{3}$ Institut für Klinikhygiene, Medizinische Mikrobiologie und Klinische Infektiologie, Universitätsinstitut der Paracelsus Medizinischen Privatuniversität, Nürnberg, Germany
\end{abstract}

The authors describe the case of a 40-year-old man suffering from an epidural abscess in the thoracic spine due to a rarely isolated pathogen, Streptobacillus moniliformis, the causative agent of rat bite fever. Besides diffuse abdominal pain, ataxia, paresthesia, hypesthesia, and enhanced reflexes of the lower extremities, the patient suffered from a decreased sensation of bladder filling. His history was also positive for a rat bite 6 weeks earlier. Magnetic resonance imaging showed an epidural, space-occupying lesion compressing the spinal cord at the vertebral levels of T6-8. Neurosurgery revealed an epidural abscess, which was drained via laminectomy (T-7) and excision of the ligamentum flavum (T6-8). The etiological agent $S$. moniliformis was identified by $16 S$ rRNA-based polymerase chain reaction and sequencing as well as by culture and mass spectrometry. Treatment with penicillin $\mathrm{G}$ led to complete resolution of the abscess and clinical recovery of the patient, who regained his bladder-filling sensation and free walking ability.

This case demonstrates that careful attention to the patient's history is essential in suspecting unusual bacterial pathogens as the cause of an epidural abscess and initiating the optimal diagnostic procedure and antimicrobial therapy.

https://thejns.org/doi/abs/10.3171/2016.12.SPINE161042

KEY WORDS epidural abscess; Streptobacillus moniliformis; rat bite; zoonosis; infection

$\mathrm{S}$ treptobacillus moniliformis belongs to the class of Fusobacteriia and is a member of the family Leptotrichiaceae. It is a fastidious, facultatively anaerobic, nonmotile, gram-negative, rod-forming bacterium with a variable morphology. The worldwide reservoirs of $S$. moniliformis are domestic and feral rats or other rodents. ${ }^{8} 12$ Recent studies have yielded rates of colonization with S. moniliformis between $17 \%$ and $92 \%$ in the nasopharyngeal tract of wild urban rats. ${ }^{11,14}$ Humans can become infected with $S$. moniliformis via several routes. Rat bite fever usually results from the bite or scratch by a rat or exposure to rodent excretions (for example, urine or saliva). Indirect transmission of the pathogen has been suggested for patients who did not report direct contact with rats but instead had a history of interactions with other domestic animals such as cats, dogs, or roosters. ${ }^{2,7,18}$ Rat bite fever that was due to the oral uptake of food or water contaminated by the excrement of rodents has been termed "erythema arthriticum epidemicum," or "Haverhill fever," named after the site of a large outbreak in the United States. ${ }^{8,12}$ Both the classic rat bite fever and the Haverhill fever are characterized by the sudden onset of fever, chills, pharyngitis, vomiting, skin rashes (maculopapular, petechial, or purpuric; affecting the extremities, predominantly the hands and feet), and arthralgias. $.^{19} \mathrm{Un}-$ like in another form of rat bite fever that is caused by an

ABBREVIATIONS CRP = C-reactive protein; MALDI-TOF = matrix-assisted laser desorption ionization with time of flight; $\mathrm{PCR}=$ polymerase chain reaction . SUBMITTED August 29, 2016. ACCEPTED December 9, 2016.

INCLUDE WHEN CITING Published online April 21, 2017; DOI: 10.3171/2016.12.SPINE161042.

* Drs. Hammer and Wolff contributed equally to this work and share first authorship. 


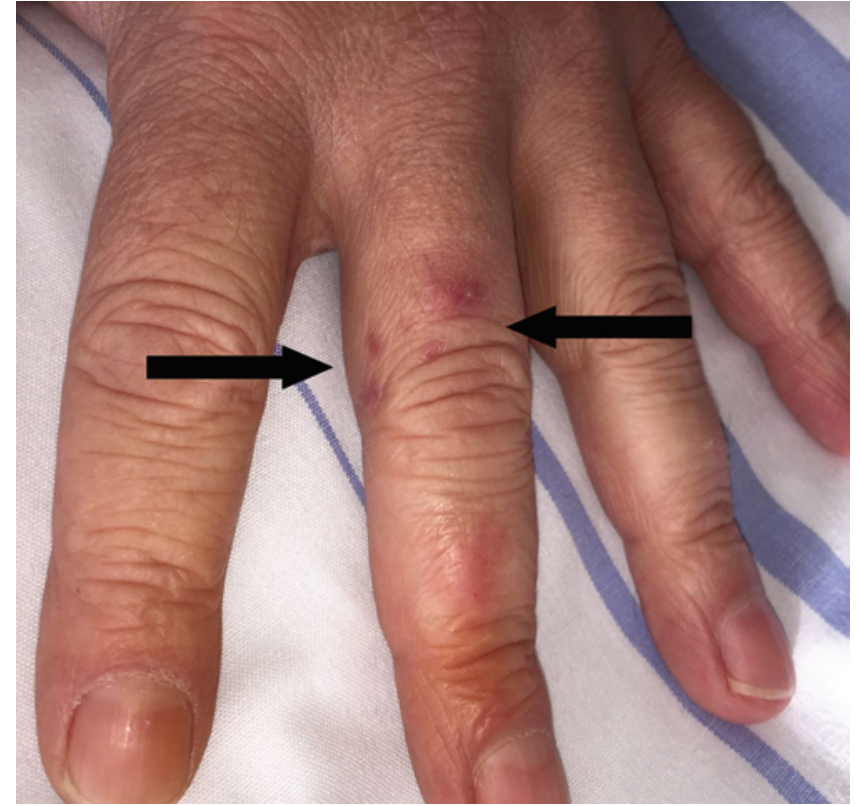

FIG. 1. Clinical condition of the site of the rat bite at the middle finger of the left hand (arrows) of the patient as detected at Day 11 of the hospital stay. Figure is available in color online only.

unrelated bacterium (Spirillum minus) and seen mostly in Asia, patients with acute streptobacillosis in general do not present with a persistent skin lesion resulting from the bite. ${ }^{12}$ Without therapy, the mortality rate ranges from $7 \%$ to $13 \% .{ }^{8,12}$ However, following appropriate antibiotic therapy, rat bite fever has a good prognosis., ${ }^{3,19}$ Severe complications of rat bite fever are endocarditis, spread of the pathogen into organs leading to abscess formation (for example, brain abscess), myocarditis, respiratory failure, and overwhelming septicemia., $3,6-8,12,15,19$ Frequently, children are affected. ${ }^{19}$ S. moniliformis infections of the human spine can occur in the form of an epidural abscess, spondylodiscitis, or a psoas abscess and have only rarely been reported. ${ }^{2,7}$ We present what we believe is the first case of an epidural abscess caused by $S$. moniliformis located in the thoracic spine.

\section{Case Report}

History and Examination

A 40-year-old man was admitted to the Department of Gastroenterology complaining of diffuse abdominal pain. Initial diagnostic imaging of the abdomen (ultrasonography, radiography) showed no pathological findings except for a bulging urinary bladder indicative of a decreased sensation of bladder filling. Neurological examination and further questioning of the patient revealed a paresthesia and hypesthesia distal to dermatome T-10, which had started 10 days earlier. Furthermore, enhanced reflexes of the lower extremities and ataxia were detected, whereas motoric functions were intact except for moderate impairment in elevation of the left foot. The patient also reported that his girlfriend's pet rat had bitten his left middle finger 6 weeks prior to the onset of his current complaints (Fig. 1). The wound had not been seen by a medical doctor and
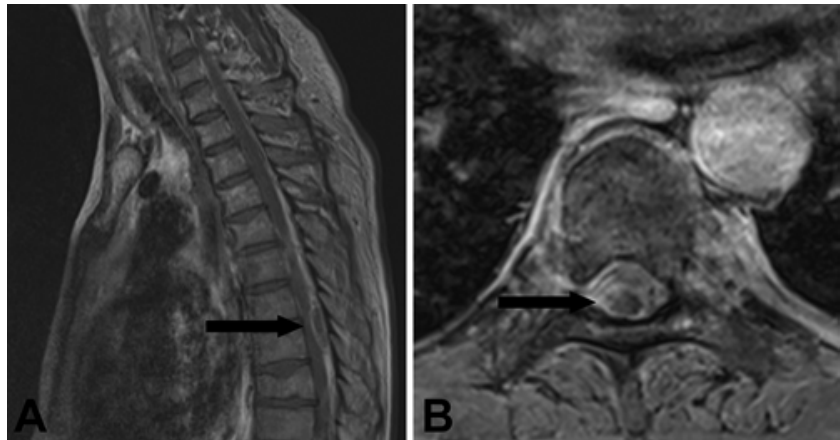

FIG. 2. Sagittal (A) and axial (B) MR images showing an intraspinal, epidural, space-occupying lesion with gadolinium contrast enhancement (arrows) at the T6-8 vertebral levels. Note the compression of the spinal cord.

had healed on its own. Fever, arthralgia, or exanthema had never developed after the rat bite.

Peripheral blood analysis on the day of admission was remarkable for prominent leukocytosis $(17,000 / \mu \mathrm{l})$ and an elevated level of the C-reactive protein (CRP; $20.3 \mathrm{mg} / \mathrm{dl}$, normal $<0.5 \mathrm{mg} / \mathrm{dl}$ ), whereas the patient's body temperature was normal $\left(37.2^{\circ} \mathrm{C}\right)$. Magnetic resonance imaging revealed an epidural, space-occupying lesion at the T6-8 vertebral levels, which showed gadolinium enhancement and compression of the spinal cord (Fig. 2).

\section{Operation and Microbiological Diagnosis}

The suspected epidural abscess was drained microsurgically via laminectomy of T-7 and excision of the ligamentum flavum at the level of T6-8. Multiple intraoperatively obtained samples of the epidural abscess were preserved for microbiological analyses.

The microbiological diagnosis was initially made by $16 \mathrm{~S}$ rRNA molecular testing of the epidural abscess sample. The DNA was extracted with the High Pure PCR Template Extraction Kit (Roche Diagnostics) according to the manufacturer's instructions. Partial 16S rRNA gene (463-bp fragment) was amplified using the Mastermix 16S Complete Kit (Molzym). Sequencing showed 100\% identity with S. moniliformis DSM 12112 (accession number NR_074449.1).

The abscess material was also plated on sheep blood agar, chocolate agar, and anaerobic agar. After 7 days of culture, one colony grew on blood agar at $37^{\circ} \mathrm{C}$ and under aerobic conditions with $5 \% \mathrm{CO}_{2}$. The bacterial isolate was identified as $S$. moniliformis by matrix-assisted laser desorption ionization with time of flight (MALDITOF, Bruker) mass spectrometry and subcultured on sheep blood agar and in serum-supplemented thioglycolate broth. S. moniliformis did not grow on chocolate agar; tested negative for catalase, indole, and oxidase; exhibited a cotton puff ball-like appearance in thioglycolate broth (Fig. 3); and showed the typical pleomorphic microscopic morphology upon Gram staining (not shown). All of these microbiological features are characteristics of $S$. moniliformis.

Antibiotic susceptibility testing using the agar diffusion methodology showed susceptibility to penicillin, cefuroxime, ceftriaxone, meropenem, gentamicin, moxifloxacin, 


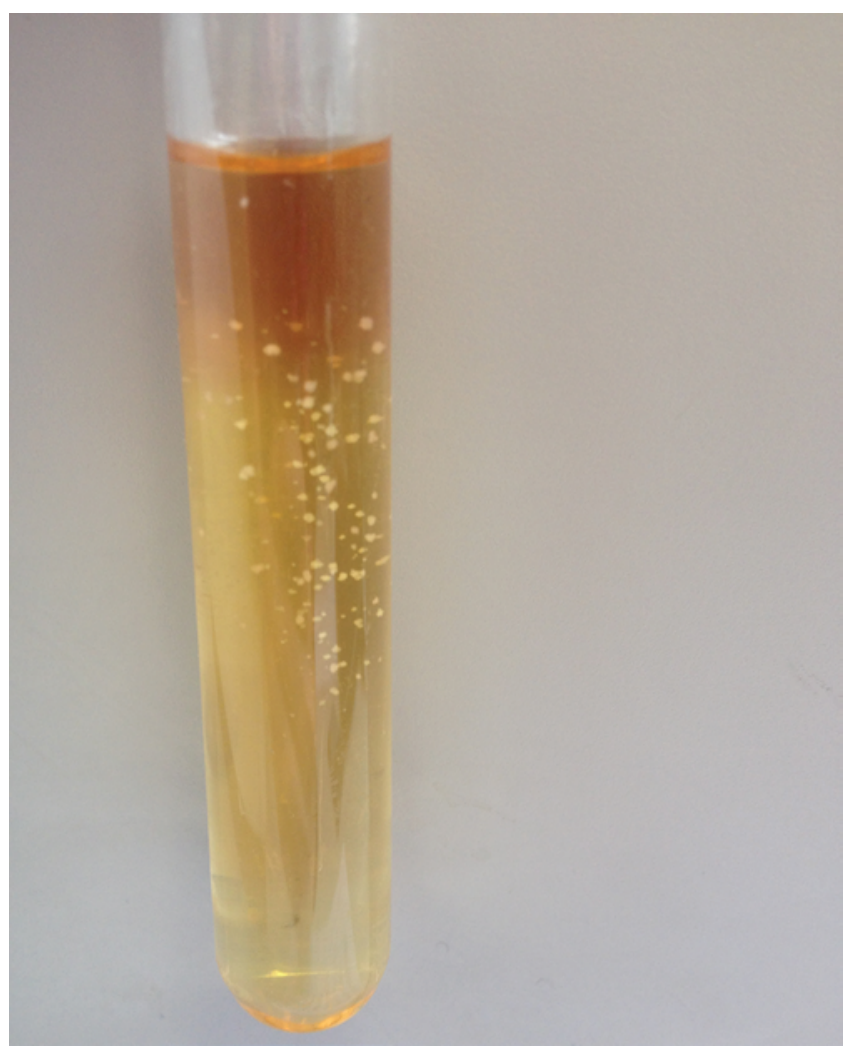

FIG. 3. S. moniliformis forming "cotton puff balls" after 2 days of incubation in thioglycolate broth containing bovine serum. Figure is available in color online only.

clindamycin, vancomycin, rifampicin, and tetracycline. The bacilli tested resistant to sulfamethoxazole-trimethoprim (co-trimoxazole) and erythromycin.

\section{Antibiotic Treatment and Further Postoperative Course}

Following the diagnosis of $S$. moniliformis by polymerase chain reaction (PCR), the antibiotic therapy was immediately switched from the empirical intravenous administration of ceftriaxone and metronidazole to the intravenous administration of penicillin $G$ for 2 weeks. This was followed by oral penicillin $\mathrm{V}$ for an additional 4 weeks. Three weeks after the neurosurgical intervention, blood analysis showed a normal leukocyte count $(7500 / \mu \mathrm{l})$ and a CRP value $<0.5 \mathrm{mg} / \mathrm{dl}$.

Control MRI was performed 2 and 6 months after surgery (Fig. 4). The epidural abscess and spinal cord compression were no longer detectable. However, T2-weighted sequences and short-tau inversion recovery (STIR) sequences revealed discrete signs of myelopathy.

The patient was transferred to a rehabilitation center where his clinical condition further improved. He regained bladder-filling sensation, and free walking at a quick pace became possible. However, he still reported reduced sensibility in his legs as well as gait and standing ataxia. A moderate paresis of the left toe also remained.

\section{Discussion}

To the best of our knowledge, the present report is only

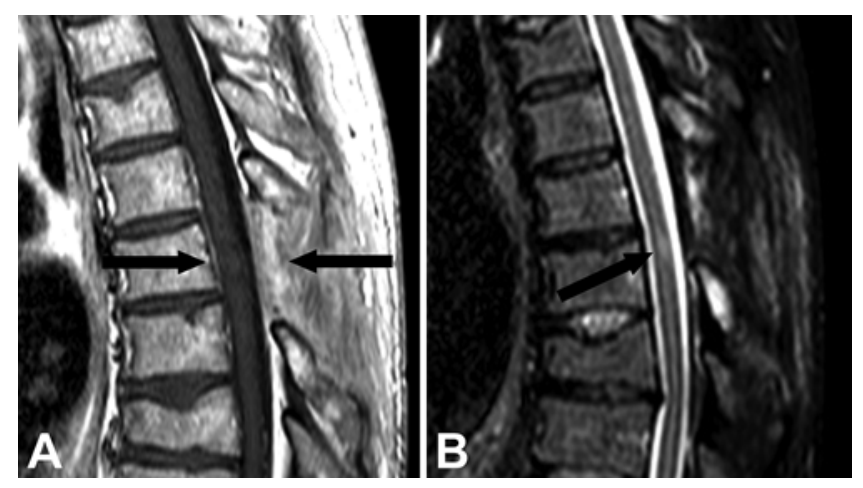

FIG. 4. A: Sagittal MR image obtained at 2 months after surgery, showing no indications of spinal cord compression. The epidural abscess was no longer detectable (arrows). B: Short-tau inversion recovery (STIR) sequence revealed discrete signs of myelopathy (arrow).

the second case in the literature in which S. moniliformis has been identified as the cause of an epidural abscess. While this zoonotic pathogen is generally regarded as a rare cause of infection, the true incidence of $S$. moniliformis infections is not known because of the difficulties in culturing the bacterium, the nonspecific symptoms of the disease, and uncertainties regarding rat exposure and the route of infection. ${ }^{2,3,15}$ In cases of proven rat bite, the risk of infection is estimated to be approximately $10 \% .{ }^{8}$ Over the past decades, cases of rat bite fever have been mostly associated with exposure to wild rat populations. Given the increasing popularity of keeping rats as domestic pets, the risk of rat bite fever rises, especially for children. Furthermore, pet store workers and laboratory technicians are at risk for $S$. moniliformis infections if the animals are not specifically pathogen free. ${ }^{2,8}$ The patient in the featured case reported that he had cared for his girlfriend's pet rat and was bitten 6 weeks before the onset of his symptoms. It is remarkable that the spinal epidural abscess (and the respective neurological complications) developed in the absence of the classic symptoms and signs of rat bite fever. It is important to note the publication of cases of rat bite fever in which there was no evidence of bite injuries by rats or other wild or domestic animals. In several instances, social deprivation, living on a farm, keeping cats and dogs, or injuries unrelated to animals were the only risk factors in the patient history. ${ }^{8,15,16,18}$

The diagnosis of rate bite fever is a challenge due to the lack of pathognomonic clinical signs and symptoms and the possible failure to detect $S$. moniliformis by routine blood cultures. Most of the standard aerobic blood culture systems contain concentrations of sodium polyanethol sulfonate as an anticoagulant that inhibits the growth of $S$. moniliformis and can result in a falsely negative blood culture. ${ }^{2,8}$ In the case of positive cultures, the examiner must be aware of the variable microscopic appearance and colony morphology. The gram-negative rods are often arranged in long tangled chains and filaments with bulbar swellings. ${ }^{8}$ Cell wall-containing wild-type $S$. moniliformis will form convex colonies or cotton puff balls in serum containing solid or liquid medium, respectively. In aged cultures, cell wall-deficient, nonpathogenic L-phase bacteria can occur, appearing like fried-egg colonies. ${ }^{8}$ In 
our case, blood cultures were taken upon patient admission to our hospital but remained negative. Instead, rapid diagnosis was achieved by molecular analysis (PCR and sequencing) of the drained epidural abscess.

S. moniliformis is almost universally susceptible to penicillin $\mathrm{G}$, which is the treatment of choice for rat bite fever. If endocarditis is present, penicillin $G$ is usually combined with gentamicin or streptomycin. Other antibiotics such as cephalosporins, tetracyclines, and aminoglycosides have also been reported to be effective in rat bite fever. ${ }^{2,8,19}$ The $S$. moniliformis isolate from our patient was fully susceptible to all tested antibiotics except for erythromycin and co-trimoxazole.

In addition to the present case, there is only one other report on a patient with an epidural abscess caused by $S$. moniliformis in the medical literature. ${ }^{2}$ In that patient, the abscess was located between the L- 4 and S-1 vertebrae, caused fever and decreased sensation of bladder filling, and was successfully treated with ceftriaxone. The microbiological diagnosis was made by culture of the pathogen from the abscess fluid, which was placed in a blood culture bottle, as well as by retrospective 16S rRNA PCR analysis of the primary specimen from the epidural abscess. The definitive diagnosis took 21 days because MALDI-TOF analysis for the identification of the cultured bacteria, which we used, was not yet available and because PCR analysis was not initially performed. Together, the 2 cases highlight the importance of molecular methods for routine microbiological diagnostics, emphasize the power of MALDI-TOF analysis of cultured bacteria (provided the underlying databases are constantly updated), and illustrate the success of combined surgical and antibiotic treatment.

Spinal epidural abscesses occur in up to $75 \%$ of cases in the thoracolumbar spine; approximately $50 \%$ are ventrally located. The most frequently detected bacteria are Staphylococcus aureus and Streptococcus species. ${ }^{17}$ Spinal epidural abscesses usually result from locally introduced bacteria (for example, via paravertebral or peridural injections), from the contiguous spread of a neighboring infection site (for example, spondylodiscitis or psoas abscess), or from the hematogenous dissemination of a distant infectious focus (for example, skin infection, dental infection, infected venous catheter, intravenous drug abuse, or endocarditis). ${ }^{4}$ As illustrated by our case, animal bites bear the risk of the inoculation of unusual bacteria, ${ }^{1}$ which subsequently can systemically spread and cause distant infections such as an epidural abscess. Our patient's history of a rat bite was instrumental in our suspecting a rare pathogen and initiating a molecular diagnostic approach, which also covers bacteria that are difficult to grow.

If the clinical and laboratory parameters of a patient suggest the presence of an epidural abscess, gadoliniumenhanced MRI of the spine should be immediately performed to ascertain the diagnosis and to plan the neurosurgical approach. ${ }^{5}$ Cases treated with early surgery typically achieve better outcomes than cases treated without surgery. ${ }^{9}$ Especially in patients who are older than 65 years, suffer from diabetes, are infected with methicillin-resistant Staphylococcus aureus, or show neurological deficits, nonsurgical medical management is not recommended..$^{13}$
Moreover, early surgery helps to avoid the development of sepsis, a leading cause of death. ${ }^{10}$

Apart from open microsurgery and antibiotics, percutaneous endoscopic debridement as well as percutaneous lavage and drainage have recently been tested as new therapies for cases of spondylitis. ${ }^{20,21}$ These methods certainly allow one to obtain adequate samples for microbiological diagnostics and can support the remission of neurological symptoms. Furthermore, we suggest that these approaches may also be suitable for treatment of paraspinal abscesses, for example, in patients who will not tolerate more invasive surgery. ${ }^{20,21}$ The percutaneous approach may also be feasible for the management of epidural abscesses.

\section{Conclusions}

In summary, after the occurrence of a spinal abscess following an animal bite, involved microbiology, neurology, and neurosurgery specialists should be aware of the possibility of rare pathogens such as $S$. moniliformis as causative agents. In these cases, rapid PCR analysis of intraoperatively obtained specimens can help to overcome the diagnostic limitations of conventional blood cultures.

\section{References}

1. Abrahamian FM, Goldstein EJ: Microbiology of animal bite wound infections. Clin Microbiol Rev 24:231-246, 2011

2. Addidle M, Pynn J, Grimwade K, Giola M: Epidural abscess caused by Streptobacillus moniliformis. J Clin Microbiol 50:3122-3124, 2012

3. Brown CM, Tsai G, Sanchez-Flores X: Oh rats! Fever, rash and arthritis in a young woman. BMJ Case Rep 2015:bcr201521240, 2015

4. Darouiche RO: Spinal epidural abscess. N Engl J Med 355:2012-2020, 2006

5. DeFroda SF, DePasse JM, Eltorai AE, Daniels AH, Palumbo MA: Evaluation and management of spinal epidural abscess. J Hosp Med 11:130-135, 2016

6. Dijkmans BA, Thomeer RT, Vielvoye GJ, Lampe AS, Mattie H: Brain abscess due to Streptobacillus moniliformis and Actinobacterium meyerii. Infection 12:262-264, 1984

7. Dubois D, Robin F, Bouvier D, Delmas J, Bonnet R, Lesens $\mathrm{O}$, et al: Streptobacillus moniliformis as the causative agent in spondylodiscitis and psoas abscess after rooster scratches. J Clin Microbiol 46:2820-2821, 2008

8. Elliott SP: Rat bite fever and Streptobacillus moniliformis. Clin Microbiol Rev 20:13-22, 2007

9. Epstein NE: Timing and prognosis of surgery for spinal epidural abscess: a review. Surg Neurol Int 6 (Suppl 19):S475S486, 2015

10. Epstein NE: What are we waiting for? An argument for early surgery for spinal epidural abscesses. Surg Neurol Int 6 (Suppl 19):S504-S507, 2015

11. Firth C, Bhat M, Firth MA, Williams SH, Frye MJ, Simmonds P, et al: Detection of zoonotic pathogens and characterization of novel viruses carried by commensal Rattus norvegicus in New York City. MBio 5:e01933-14, 2014

12. Gaastra W, Boot R, Ho HT, Lipman LJ: Rat bite fever. Vet Microbiol 133:211-228, 2009

13. Kim SD, Melikian R, Ju KL, Zurakowski D, Wood KB, Bono CM, et al: Independent predictors of failure of nonoperative management of spinal epidural abscesses. Spine $\mathbf{J}$ 14:1673-1679, 2014

14. Kimura M, Tanikawa T, Suzuki M, Koizumi N, Kamiyama $\mathrm{T}$, Imaoka K, et al: Detection of Streptobacillus spp. in feral 
rats by specific polymerase chain reaction. Microbiol Immunol 52:9-15, 2008

15. Kondruweit M, Weyand M, Mahmoud FO, Geissdörfer W, Schoerner C, Ropers D, et al: Fulminant endocarditis caused by Streptobacillus moniliformis in a young man. J Thorac Cardiovasc Surg 134:1579-1580, 2007 (Erratum in J Thorac Cardiovasc Surg 135:770, 2008)

16. Regnath T, Kurb N, Wolf M, Ignatius R: [Rat-bite fever-two cases of infection with Streptobacillus moniliformis within two months.] Dtsch Med Wochenschr 140:741-743, 2015 (Ger)

17. Shweikeh F, Saeed K, Bukavina L, Zyck S, Drazin D, Steinmetz MP: An institutional series and contemporary review of bacterial spinal epidural abscess: current status and future directions. Neurosurg Focus 37(2):E9, 2014

18. Torres L, López AI, Escobar S, Marne C, Marco ML, Pérez M, et al: Bacteremia by Streptobacillus moniliformis: first case described in Spain. Eur J Clin Microbiol Infect Dis 22:258-260, 2003

19. Wullenweber M: Streptobacillus moniliformis-a zoonotic pathogen. Taxonomic considerations, host species, diagnosis, therapy, geographical distribution. Lab Anim 29:1-15, 1995

20. Yang SC, Chen WJ, Chen HS, Kao YH, Yu SW, Tu YK: Extended indications of percutaneous endoscopic lavage and drainage for the treatment of lumbar infectious spondylitis. Eur Spine J 23:846-853, 2014
21. Yang SC, Fu TS, Chen HS, Kao YH, Yu SW, Tu YK: Minimally invasive endoscopic treatment for lumbar infectious spondylitis: a retrospective study in a tertiary referral center. BMC Musculoskelet Disord 15:105, 2014

\section{Disclosures}

The authors report no conflict of interest concerning the materials or methods in this study or the findings specified in this paper.

\section{Author Contributions}

Conception and design: Hammer, Steiner, Bogdan. Acquisition of data: Hammer, Wolff, Schrey, Ziegler, Bogdan. Analysis and interpretation of data: Hammer, Wolff, Geißdörfer, Bogdan. Drafting the article: Hammer, Wolff, Bogdan. Critically revising the article: Hammer, Wolff, Geißdörfer, Bogdan. Reviewed submitted version of manuscript: Hammer, Wolff, Geißdörfer, Bogdan. Approved the final version of the manuscript on behalf of all authors: Hammer. Administrative/technical/material support: Steiner, Bogdan. Study supervision: Steiner, Bogdan.

\section{Correspondence}

Alexander Hammer, Department of Neurosurgery, Paracelsus Medical University, Breslauer Straße 201, 90471 Nürnberg, Germany. email: alexander.hammer@klinikum-nuernberg.de. 